livraisons

d'Histoire

de l'Architecture

\section{Livraisons de l'histoire de l'architecture}

14 | 2007

Piscines

\title{
De la « fresque primitive » au mur « où tout est ornement » : les contributions d'André Lhôte à une théorie de l'art mural (1920-1940)
}

From 'primitive frescoes' to a mural aesthetic where 'ornament is everything': André Lhote's contributions to a theory of mural art Von primitiven Fresken zur Auffassung der Wand als Ornament : der Beitrag André Lhôtes zu einer Therorie der Wandmalerei

Jean-Roch Bouillier

\section{OpenEdition}

Journals

Édition électronique

URL : http://journals.openedition.org/lha/434

DOI : $10.4000 /$ /ha. 434

ISSN : 1960-5994

Éditeur

Association Livraisons d'histoire de l'architecture - LHA

Édition imprimée

Date de publication : 10 décembre 2007

Pagination : 113-123

ISSN : 1627-4970

Référence électronique

Jean-Roch Bouillier, « De la « fresque primitive » au mur « où tout est ornement » : les contributions d'André Lhôte à une théorie de l'art mural (1920-1940) », Livraisons de l'histoire de l'architecture [En ligne], 14 | 2007, mis en ligne le 10 décembre 2009, consulté le 01 mai 2019. URL : http:// journals.openedition.org//ha/434 ; DOl : 10.4000//ha.434

Ce document a été généré automatiquement le 1 mai 2019.

Tous droits réservés à l'Association LHA 


\section{De la « fresque primitive » au mur «où tout est ornement »: les contributions d'André Lhôte à une théorie de l'art mural (1920-1940)}

From 'primitive frescoes' to a mural aesthetic where 'ornament is everything': André Lhote's contributions to a theory of mural art Von primitiven Fresken zur Auffassung der Wand als Ornament : der Beitrag André Lhôtes zu einer Therorie der Wandmalerei

Jean-Roch Bouillier

La vitalité de la peinture murale dans la France du début du XX $\mathrm{X}^{\mathrm{e}}$ siècle est si sensible dans la pratique de nombreux artistes et dans les écrits contemporains sur l'art qu'on peut discerner, à cette époque, l'émergence d'une véritable théorie de l'art mural ${ }^{1}$.

2 André Lhote (1885-1962), figure marquante du mouvement cubiste et des tendances du « rappel à l'ordre ", à la fois peintre, auteur et pédagogue, prend part à ce mouvement de théorisation, qui tend à définir un cadre autour de pratiques cherchant souvent à mêler référence à la tradition artistique et renouvellement moderne.

Lhote pratique la peinture murale, tout d'abord, en tant que peintre, dans des chantiers privés $^{2}$ puis, comme pour beaucoup de ses contemporains, à l'occasion de l'Exposition universelle de 1937, dans deux grandes compositions, intitulées La houille - les fours à coke et Le gaz, effectuées pour la salle de la chimie organique, au Palais de la découverte. L'année suivante, il réalise une nouvelle peinture murale, sur le thème de l'agriculture industrielle, pour l'École des arts et métiers de Paris ${ }^{3}$. Il y revient après la deuxième guerre mondiale, en 1955, pour une grande composition sur la Gloire de Bordeaux, à la faculté de médecine de cette ville4.

4 Mais c'est surtout en tant que théoricien qu'André Lhote démontre sa foi dans la valeur de l'art mural, qu'il exprime sa combativité en faveur de sa pratique et qu'il fait preuve d'une grande fidélité à ce thème, au fil des centaines d'articles qu'il publie, entre 1919 et 
1940. La régularité de son recours à cette notion, la mise en place d'un vocabulaire spécifique et la place réservée à la question du mur, dans son élaboration d'une théorie de l'art, autorisent à parler, dans le cadre de l'étude de ses écrits, de «théorie de l'art mural $»^{5}$.

Cette théorie apparaît de différentes manières : tout d'abord, à travers ses commentaires sur les œuvres d'art héritées du passé ou celles de ses contemporains ; à travers ses leçons de pédagogue et ses conseils pour la pratique de la peinture et enfin, à travers ses commentaires sur l'architecture de son temps.

\section{La fresque primitive}

André Lhote exprime une conception mythique de la "fresque primitive ", comme en témoigne une lettre sur ce sujet, adressée à son ami Jacques Rivière (1886-1925), dès 1909 :

Pour expliquer l'idée fort simple que je me fais du tableau, il faudrait recourir à un procédé lyrique dans le genre de Développement de l'Église [de Paul Claudel]. Il faudrait partir de cette histoire de la fille du potier qui traça sur le mur le contour de l'ombre du visage de son fiancé - le modèle parti, on imagina de signifier l'opacité absente par un sommaire et précis remplissage. Quant à la paroi de la demeure succède le mur du temple, il s'agit toujours de décorer la nudité stable et crue. L'habitation est pleine de calme et de rectitude. L'artiste habille le mur de contours plats et tranquilles, adéquats à la sévérité des lignes architecturales. Mais de nos jours, voici qu'aux murs grimpent les meubles dont le déplacement incessant en abolit la totale et harmonieuse figure. Leur surface ne peut supporter d'empreinte durable. Alors, imaginativement, on en découpe des parcelles que l'on orne et que l'on réapplique pour un instant: le tableau qu'encadrent des baguettes, au lieu des boiseries le mur6.

Dans ce passage, Lhote dit tout de sa conception du mur et du tableau. Il n'est pas surprenant de trouver dans ses articles de critique d'art, à partir de 1919, des références régulières à la «fresque primitive » en tant que source originelle de l'art, dont il ne faut oublier ni l'essence ni les enseignements. En 1920, il reprend, à propos de Paul Cézanne (1839-1906), l'idée selon laquelle le tableau de chevalet est le succédané moderne du mur :

Cézanne, après avoir utilisé uniquement les richesses dangereuses de l'arsenal romantique [...] dut y renoncer pour redevenir en quelque sorte son propre primitif. Comprenant tout à coup les exigences du mur dont la toile est le symbole, il retrouva [...] le langage plastique dans sa limpidité originelle ${ }^{7}$.

Le fait que Lhote invoque ici Cézanne, comme dans presque chacun de ses textes, mais que parallèlement, il ne fasse allusion à Pierre Puvis de Chavannes (1824-1898) dans aucun de ses écrits montre que sa conception de l'art mural est plus théorique que véritablement centrée sur les seules peintures murales. La capacité d'un artiste à « retrouver le mur» devient alors, pour Lhote, un critère parmi d'autres pour juger la qualité de son œuvre, quel qu'en soit le support : Gauguin, au moins, se posa les problèmes suivants, à résoudre par des moyens qu'il croyait strictement plastique: 1 . retrouver le mur [...] 2. ramener en larges nappes l'intensité colorée dispersée en touches brisées par l'impressionnisme ${ }^{8}$.

Ce regard critique vaut aussi pour les œuvres contemporaines et, en tout premier lieu, pour les peintures cubistes. En 1924, devant définir les caractéristiques de ces dernières, il 
insiste sur leur capacité à épouser la surface de la toile ou le plan du mur et à s'opposer ainsi à l'idée du tableau conçu, depuis Leon Battista Alberti (1406-1472), comme une fenêtre illusoire. Ce souci de l'aplat rapproche, selon lui, les cubistes de l'art des primitifs ${ }^{9}$ - Quatre ans plus tard, Lhote parle, à propos de son propre travail de peintre, de la nécessité de remonter aux sources et de s'inspirer des disciplines techniques les plus éprouvées: celles des Primitifs, amenant à utiliser des teintes plates, localisées, appliquées par couches successives, selon la technique du peintre en bâtiment. Le mur, d'où sortit la fresque originelle et dont le tableau n'est qu'une réduction est toujours suggéré, d'après lui, dans l'œuvre du Primitif, sans qu'il n'y ait jamais d'échelonnement en profondeur. La Renaissance et l'impressionnisme ayant bouleversé cela, il convient, à travers des habitudes nouvelles, de retrouver la maîtrise ancienne ${ }^{10}$.

À la fin des années 1930, plusieurs articles montrent que Lhote conserve, près de trente ans après sa lettre à Jacques Rivière, citée ci-dessus, sa vision mythique de l'art mural. Rendant compte de l'exposition des Chefs-d'œuvre de l'art français, organisée au PetitPalais, en 1937, il clame son admiration devant les enluminures, les tableaux, les fresques, les tapisseries et les vitraux médiévaux : «C'est que derrière la tapisserie et le tableau est le mur. [...] tout est ornement car l'ornement est ce qui parle le mieux, le plus directement aux sens et à l'esprit ${ }^{11}$. »

Il parle ensuite du "passage de l'ornement pur à l'ornement incarné ", à travers les œuvres de Jean Fouquet (c. 1425-1480), de Nicolas Froment (c. 1435-1483) et du Maître de Moulins (seconde moitié du XV ${ }^{e}$ siècle). Mais « avec le XVII ${ }^{e}$ siècle s'ouvre l'ère du fruit défendu ", c'est-à-dire celle de la lumière qui fait saillir les plans, au lieu de les niveler, et de l'objet qui penche «dangereusement en dehors de la toile $»^{12}$. Quant au XVIII ${ }^{\mathrm{e}}$ siècle, c'est une « monstrueuse époque où tout le monde, même les portraitistes, peignait dans le vide $»^{13}$. Heureusement, l'art de Jean-Auguste-Dominique Ingres (1780-1867) est celui du « rétablissement sur le plan, à la façon des Primitifs, de gestes qui tendent à se déployer en profondeur $\aleph^{14}$. Lhote renchérit un an plus tard dans le même sens :

Bien entendu, c'est à ses débuts que la tapisserie produisit ses chefs d'œuvre les plus purs. Ypodame ravie par les Centaures, pièce sortie des ateliers de Tournai au $\mathrm{XV}^{\mathrm{e}}$ siècle, constitue un spécimen parfait de cet art, qui est soumis aux lois tyranniques de l'à-plat mural, de la superposition des motifs, de la réduction des gammes colorées, et du dessin ornemental, c'est-à-dire synthétique et géométrisé. [...] Dans les tapisseries du XVII siècle, au contraire, nous sommes entraînés dans un tourbillon général ${ }^{15}$.

La conception d'un "art mural », chez Lhote, n'est donc pas restreinte aux seules peintures murales et à tout ce qui pourrait s'y apparenter. Elle est beaucoup plus large et trouve sa naissance dans son observation de l'histoire de l'art. Les textes cités permettent à la fois de souligner la permanence de ce concept, chez l'artiste-auteur, des années 1900 aux années 1930, sa forte dimension mythique et son importance pour juger les œuvres. Celles des XVII et ${ }^{\mathrm{XVIII}}{ }^{\mathrm{e}}$ siècles ne méritent ainsi, selon lui, que peu d'attention, en raison de leur éloignement de la technique « primitive».

\section{Peinture murale et tableau de chevalet}

17 Si André Lhote considère que le tableau de chevalet est l'héritier légitime du mur, il ne pense pas que tout tableau doit receler une anamnèse de ce dernier. Il met en balance, dès 1924, des artistes spécialisés dans la peinture murale et d'autres qui se consacrent aux tableaux de chevalet : 

quelques mois que Rubens en cinq années - ou bien sont-ce ces jeunes peintres [...] ? Celui-ci se collète avec les sujets les plus nombreux et les plus dangereux; ceux-là, à la suite de Braque et de Picasso, réduisent insensiblement la peinture à une spéculation purement technique sur des sujets de plus en plus minuscules ${ }^{16}$.

Au fil des années, le mot «technique " prend sous la plume de Lhote un sens plus précis. Chaque artiste doit, selon lui, adopter un langage différent et une technique spécifique en fonction du support choisi : la peinture murale ou le tableau de chevalet ou encore le croquis sur le motif... Il sous-entend ainsi que la peinture murale ou le tableau de chevalet doivent donner lieu à des approches différentes. Ce souci de cohérence entre un support, un outil et une certaine approche esthétique permet au critique de juger, dans les années 1930, l'adéquation entre la technique choisie et ce qui est exprimé :

On pouvait constater devant leurs œuvres [Friesz, Dufy, Segonzac], que les meilleures aquarelles sont celles qui se différencient le plus de la peinture à l'huile. [...] On s'étonne de voir des peintres obtus, insensibles aux nécessités de leur technique. [...] Peindre à l'aquarelle le même objet qu'à l'huile est une monstruosité ${ }^{17}$.

Chassériau, " conscient d'être soumis à des modes d'expression opposés, les assume avec courage et en légitime l'emploi par la destination même des surfaces peintes. Lorsque le goût des arrangements calmes le hante, c'est à la décoration du mur qu'il l'applique ; ce sont alors les belles fresques apaisées de la cour des Comptes, ou même de grands tableaux comme Les Troyennes, qui sont des tableaux-fresques, en mal d'architecture. Lorsque au contraire la passion du mouvement le prend, c'est au tableau de chevalet qu'il la confie : celui-ci, qui ne s'accroche au mur que provisoirement, que l'on peut placer sur un chevalet ou tenir dans les mains comme un organisme palpitant, se plie à toutes les fantaisies. [...] Chassériau adopte des conceptions picturales différentes selon qu'il s'adresse au mur ou au tableau ${ }^{18}$. »

Chassériau représente aux yeux de Lhote l'artiste le plus apte à adapter son vocabulaire. Cet exemple lui permet d'opposer des langages spécifiques: l'un convenant à la décoration du mur, considéré comme statique ; l'autre aux tableaux de chevalet, supposés dynamiques. Toujours au milieu des années 1930, André Lhote en arrive ainsi à une deuxième opposition, découlant de cette première : «Est-il possible d'atteindre l'humain à travers le décoratif? [...] Le dilemme Décoration-Expression existe depuis l'invention même de la peinture occidentale, depuis les enluminures et les fresques ${ }^{19}$. »

L'opposition mur-tableau se transforme donc en un "dilemme Décoration-Expression ", Lhote nommant enfin clairement les approches esthétiques spécifiques qu'il appelle de ses vœux depuis deux décennies. Au passage, il donne une légitimité à son idée par une pirouette généalogique, faisant remonter le décoratif-mural et l'expressif-tableau à l'époque médiévale.

\section{Leçons pour la pratique de la peinture}

Comme souvent chez André Lhote, les idées développées dans le cadre de ses articles de critique d'art se transforment en idées générales et en théories, pour être utilisées, dans un second temps, comme préceptes à transmettre. Le recours à la notion d'art mural vient ainsi justifier la nécessité, définie par l'artiste-auteur, dès 1914, de tenir compte de la bidimensionalité de la surface pour asseoir la composition, y compris dans les tableaux 
de chevalet: «Une seule loi profonde, de tous temps, régit mes tentatives : la nécessité, pour l'œuvre accrochée au mur, de soutenir un rapport avec l'architecture intérieure, que cette architecture soit déterminée par la construction même de l'édifice ou par les lignes des meubles qui le garnissent ${ }^{20}$.»

Près de dix ans plus tard, Lhote décortique encore, à des fins d'explication, les «moyens nouveaux » utilisés par les cubistes : «Ce sont : $1^{\circ}$ La composition murale, par répartition du haut en bas de la toile, des éléments plastiques, sans trou, ni perspective trompe l'œil. [...] $2^{\circ}$ La répartition rythmique des ornements géométriques, la répétition de l'angle droit et la franchise des tons locaux ${ }^{21}$.»

Et il explique à nouveau, plusieurs années après, la méthode à adopter pour atteindre tout à la fois, en peinture, une dimension décorative et une dimension murale :

Le caractère décoratif, qui convertit le tableau de chevalet en un vêtement du mur, provient d'une opération quasi magique qui consiste à faire chavirer sur la surface de la toile le spectacle (vu ou imaginé) de façon que les parties les plus éloignées de l'œil remontent et viennent s'installer au sommet de la composition, sous un ciel devenu aussi solide que le premier plan.

Le deuxième précepte mis en avant par André Lhote, en lien avec la notion d'art mural, porte sur la nécessité de tenir compte de la bidimensionalité du support dans l'utilisation de la couleur. Il débouche sur la notion de «teinte plate». La « teinte plate exigée par le mur $»^{22}$ correspond tout d'abord, chez lui, à une utilisation de la couleur qui renonce à la représentation réaliste et qui épouse, là encore, la planéité de la surface. «Les modernes un peu honnêtes et conscients, délaissant l'art perdu des glacis, ont adopté l'antique, le primitif métier de la teinte plate ${ }^{23}$. " La teinte plate correspond aussi à une approche décorative de la couleur, vis-à-vis de laquelle André Lhote n'est pas toujours bienveillant, mais à laquelle il reconnaît, dans certains cas, une valeur : « Leur technique est celle de la teinte plate, remplissant des formes qu'une ligne continue délimite entièrement. [...] Cette ligne continue, ces teintes plates, ces ornements complices ressortissent à la décoration ${ }^{24}$ . \

Les leçons tirées par Lhote de ses considérations sur l'art mural, qu'elles touchent au respect de la bidimensionalité ou à l'utilisation de la couleur, aboutissent ainsi à une définition de l'art décoratif et aux liens que ce dernier doit entretenir avec l'architecture.

\section{Liens de l'art mural avec l'architecture contemporaine}

Si André Lhote défend la dimension décorative de l'art mural, c'est d'abord pour mieux la condamner, s'il la perçoit dans des tableaux de chevalet. Le caractère ornemental ou décoratif d'une toile est, en effet, un des critères auquel il recourt régulièrement pour juger une œuvre. Comme il importe avant tout, pour Lhote, de prendre en compte le support de celle-ci, la surface murale autorise, selon lui, une approche décorative alors que le tableau de chevalet ne le permet pas : « La hâte et le calcul poussent trop d'artistes à confondre le tableau, surface où se ramassent et se concentrent le plus d'éléments possible, avec le panneau décoratif, superficiellement recouvert d'ornements empruntés au réel ${ }^{25}$.»

Le "panneau décoratif » trouve en revanche, pour Lhote, toute sa légitimité dans le contexte architectural. L'artiste-auteur interpelle vivement, dans ses écrits, pouvoirs publics et architectes pour que la construction moderne laisse une place à la décoration. 
Parlant de "l'architecture inhumaine des façades et des meubles nus ${ }^{26}$, il critique l'absence de peinture ou de sculpture dans les bâtiments contemporains :

Si l'on excepte l'admirable théâtre de Perret et l'ambassade de Mallet-Stevens, aucune nécessité profonde ne semble motiver les proportions de la plupart des bâtiments qui se parent, selon un nouveau poncif, de mensongère nuditéz ${ }^{27}$.

Pour ma part je me réserve de défendre un jour l'idée suivante : la grandeur d'une architecture se vérifie à son ornementation ${ }^{28}$.

31 Ces prises de position expliquent le conflit qui l'oppose, au milieu des années 1930, à Le Corbusier (1887-1965). André Lhote écrit, le 25 septembre 1934, une lettre de félicitations à Jacques Guenne pour son article critique sur l'Union des artistes modernes (UAM), publié dans l'Art vivant, qui ne laisse aucun doute sur ses sentiments à l'égard de l'architecte : «Il faut oser dire enfin combien nous, artistes et rêveurs, et amis justement de cette fantaisie dont vous faîtes si bien de parler, sommes fatigués de cette architecture fonctionnelle, hygiénique et parpaillotte que prône avec un manque de réserve étonnant cet enfant gâté de l'Helvétie qui a nom Le Corbuzier [sic $]^{29}$. »

Le 7 février 1935, Lhote réagit à un article de Le Corbusier, que l'auteur vient de lui envoyer, destiné à la revue Prélude $e^{30}$ :

Mon cher Le Corbuzier [sic], [...] Naturellement, je ne me comprends pas dans le nombre des «froussards, des indigents d'esprit, des faibles de vitalité, qui s'emploient avec un acharnement néfaste à détruire ce qu'il y a de plus beau dans ce pays et dans cette époque »: l'architecture de Le Corbuzier. Non, pas plus que vous n'aurez à vous comprendre parmi ces « énergumènes qui veulent nous forcer à renifler l'époque à travers les fumées d'un moteur à explosion et qui se croient seuls détenteurs de lucidité et d'enthousiasme » 31 .

Lhote poursuit sa lettre par une série de questions visant à savoir s'il accepte que la maison d'habitation se différencie extérieurement de l'usine, s'il ne se sent pas coupable de l'oubli dans lequel on tient la peinture et la sculpture (un amateur pour qui Le Corbusier a bâti une immense maison ayant dû mettre ses œuvres dans des placards), ou encore si l'on pourrait égayer les façades des " machines à habiter " $^{32}$ par des éditions de sculptures de Zadkine ou de Laurens.

Le fait que Lhote condamne la nudité des bâtiments, qu'il loue les constructions d'Auguste Perret (1874-1944), de Robert Mallet-Stevens (1886-1945) et, à l'opposé, qu'il parle du Petit-Palais comme du " temple de l'imbécillité architecturale $»^{33}$ permet de voir en Lhote l'amateur d'une architecture située entre les deux, celle précisément de l'art déco. C'est ainsi qu'il apparaît comme un des défenseurs de l'art mural et de l'ornement, dans l'entre-deux-guerres, période où ces notions se trouvent au centre des débats artistiques.

\section{Au pied du mur}

Les débats sur l'art mural donnent lieu, en 1935, à la création d'un salon de l'art mural, dont Amédée Ozenfant est président du comité technique et André Lhote, avec de nombreux autres artistes, membre du comité d'honneur ${ }^{34}$. Par ailleurs, Lhote rend compte d'une des expositions de ce Salon, en 1938. Il rappelle qu'il a été créé par SaintMaur et Schœdelin. Toutes les tendances artistiques y sont représentées, mais le cubisme et le surréalisme sont selon lui dominants. Il mentionne les envois importants de Matisse 
et de Picasso. Robert et Sonia Delaunay, Gleizes, Villon, Herbin, Power, Dyl sont fidèles à l'art abstrait. Saint-Maur, Schœdelin, Lurçat, Vérité, Survage, Kuss font des efforts " pour ré-humaniser la peinture décorative ». Léger, Freundlich, Valensi, Caroline Hill tendent vers un art décoratif pur. Enfin quelques jeunes peintres sont « séduits par l'art roman, le seul qui, à l'époque du béton armé, puisse inspirer l'art décoratif moderne ${ }^{35}$ ».

Un objectif militant n'est pas étranger à l'existence de ce Salon ${ }^{36}$. Créé deux ans avant l'Exposition de 1937, il permet à des artistes de mettre en avant leurs aptitudes à la peinture monumentale à un moment où les commandes publiques doivent se développer. La perspective de ce nouveau marché aiguise d'ailleurs le sens des revendications :

On entend des jeunes peintres enflammés par une idéologie sociale appeler de leurs vœux la fin du capitalisme, l'instauration d'un régime étatiste où ils seraient, chacun selon ses forces, chargés d'accomplir des œuvres décoratives, monumentales ou de moindre envergure pour l'État, patron et mécène équitable, généreux, mais ménager de ses munificences ${ }^{37}$.

Toute grande époque s'est chantée sur ses murs [...] Les arts muraux n'ont jamais prospéré que dans les époques où le sens collectif existait [...] L'égoïsme individualiste est déjà en régression. Les arts d'usage collectif pourront renaître quand d'un statut collectif nouveau émanera un esprit nouveau ${ }^{38}$.

Il reste cependant à observer que la cause de l'art mural, celle du retour au métier et de la réhabilitation de valeurs artistiques ancestrales est susceptible de faire l'objet d'un large consensus, illustré par les propos officiels tenus, dès 1934, avant l'arrivée du Front populaire au pouvoir, au sujet des peintures murales de l'Exposition internationale de 1937. Celles-ci devront «montrer que le souci d'art dans le détail de l'existence journalière peut procurer à chacun, quelle que soit sa condition sociale, une vie plus douce, qu'aucune incompatibilité n'existe entre le beau et l'utile ${ }^{39}$.

Il n'est pas anodin, dans ce contexte, qu'André Lhote prenne le soin, au fil de ses articles, de distinguer et de définir ce que doivent être, selon lui, le tableau de chevalet et l'art mural. Sa position sur ces questions reste toutefois prudente et modérée. Il veille justement à ne pas les politiser et à les aborder d'un point de vue technique, qui trouve toujours sa justification dans l'histoire. Contrairement à certains de ses confrères, associant revendications sociales et appels à la peinture murale, Lhote s'en tient à une analyse froide des paramètres picturaux. Alors que la référence au métier traditionnel, à l'art mural et $a$ fortiori à la fresque - en tant que technique exigeante, sous-tendant une implication physique et morale totale de l'artiste - peut avoir une connotation idéologique très forte, Lhote en retire surtout pour sa part un pur concept plastique : le mur, dont il postule, sans chercher à en faire la démonstration, qu'il est le support légitime de l'œuvre d'art et, d'autre part, une injonction, tout aussi peu démontrée : l'impératif de respecter cette bidimensionalité et de ne pas se laisser aller à la perspective. Ces deux données lui servent à étayer à bon compte ses propres théories et à exploiter la garantie de sérieux que confère l'aura de la peinture murale :

La verticalité sur l'horizontalité, correspond aux éléments architecturaux de la pièce. Les écarts entre les surfaces principales ont été calculés avec le fameux nombre d'or. Le panneau carré, impliquant le cercle, m'a incité à choisir la courbe comme rythme dominant. [...] Le peintre de chevalet peut être un joyeux drille au maintien débraillé ; le fresquiste (même s'il peint sur toile) doit, tout passionné qu'il soit, composer avec la raison ${ }^{40}$. 
43 Par ailleurs, alors que certains auteurs cherchent, à travers cette question de l'art mural, à donner une dimension collective à la création artistique, confiant à l'architecte un rôle de coordonnateur et à l'artiste un rôle d'exécutant - modèle à rapprocher de l'idéal mythique du chantier médiéval ${ }^{41}$, Lhote se pose au contraire, en tant qu'artiste-auteur, en rival de l'architecte, condamnant les constructions modernes nues et réclamant la réhabilitation du grand décor d'architecture, non pour y être inféodé mais pour que le peintre y apparaisse en bonne place ${ }^{42}$.

44 La notion d'art mural occupe une place importante dans les écrits d'André Lhote entre 1920 et 1940, période la plus riche de sa carrière d'auteur, même s'il n'a pas conçu une théorie de l'art mural à part entière comme il a élaboré, en 1939, un Traité du paysage ou, en 1950, un Traité de la figure. En effet, non seulement il met lui-même en avant cette notion mais, en outre, en tant que critique d'art, il rend compte d'une actualité où l'art mural tient une place également importante.

Après 1940, André Lhote continue à aborder cette question, d'abord dans des articles épars comme "Saint-Savin "Sixtine" française ", en 1944, à l'occasion de l'édition d'un album sur les peintures murales de Saint-Savin-sur-Gartempe ${ }^{43}$. Il y vante le dessin dynamique des six ouvriers de la Tour de Babel, qui n'est pas disproportionné mais qui serait diminué par la perspective et le clair-obscur. Il loue les vides entre les personnages, qui sont aussi expressifs que les pleins. Il ne s'agit pas, pour Lhote, de revenir aveuglément aux procédés des $\mathrm{XI}^{\mathrm{e}}$ et $\mathrm{XII}^{\mathrm{e}}$ siècles, « mais de s'y référer avec passion pour retrouver le sens perdu du rythme constructif et celui, légèrement compromis par les abstracteurs de quintessence, de l'expression, transposée, de la vie »"4. De même, en 1949, il parle encore des tentatives de Gauguin pour « faire rentrer dans l'à-plat mural » les rondeurs de l'impressionnisme. Il indique deux manières de lutter contre le trou que constitue le ciel dans le tableau: dessiner un horizon haut ou diffuser de la clarté dans le reste de la composition ${ }^{45}$.

Mais c'est surtout dans un de ses derniers ouvrages que Lhote revient longuement sur la question de la peinture murale. Après sa découverte, de 1950 à 1952 des peintures des temples en Égypte, il publie, en 1954, Les Chefs-d'œuvre de la peinture égyptienne ${ }^{46}$. Il y décrit les peintures pharaoniques et leurs liens avec la peinture moderne. Il les oppose aux peintures murales des cavernes qui, pour lui, sont inutilisables, parce que nullement ordonnées ${ }^{47}$. Il essaie au contraire de montrer que le chevauchement des plans, le faux bois, l'importance émotive des objets, la conjugaison de la face et du profil, du plan et de l'élévation se retrouvent à la fois dans la peinture égyptienne et dans le cubisme. Pour Lhote, la peinture moderne tend donc, elle aussi, vers l'à-plat mural, en raison de son goût pour «l'absolu décoratif». Ce dernier conduit, certes, à l'art abstrait. Lhote le reconnaît en même temps qu'il le déplore. En ce milieu des années 1950, la scène artistique a beaucoup évolué depuis ses premières phrases sur le mur et le tableau. Mais sa vision de l'art mural, elle, n'a pas changé. 


\section{NOTES}

1. Ce texte correspond à une communication pour le colloque La Peinture murale au début du XXe siècle en France : 1920-1940, Saint-Savin-sur-Gartempe, Centre international d'art mural, 19-21 mars 2003, dont je remercie ici les organisateurs. Je remercie également MM. Dominique Jarrassé, Jean-Michel Leniaud, Laurent Ferri et Emmanuel Luis pour leurs conseils.

2. Dans une lettre à Jacques-Émile Blanche, du 28 décembre 1934, André Lhote écrit que sa lecture du livre de Jacques-Émile Blanche, Mémoires de Joseph Perdillon a été interrompue « à cause d'une peinture murale (oui par ces temps!) qu'un architecte de province m'a commandée ». Bibliothèque de l'Institut de France, lettres d'André Lhote à M. et Mme Jacques-Émile Blanche, cote Ms 7048, feuillet 102.

3. Voir Philippe Dufieux, «Lhote et le décor monumental », dans le catalogue d'exposition André Lhote (1885-1962), Paris, Réunion des musées nationaux, Valence, musée des beaux-arts, 15 juin - 28 septembre 2003, p. 80-93.

4. Voir Françoise Garcia, «Lhote : la gloire de Bordeaux », dans le catalogue d'exposition André Lhote (1885-1962), 2003, op. cit., p. 94-99.

5. . Voir Jean-Roch Bouiller, Définir et juger l'art moderne. Les écrits d'André Lhote (1885-1962), thèse sous la direction de Mme Françoise Levaillant, directrice de recherche au CNRS, université Paris I Panthéon - Sorbonne, 2004.

6. Lettre d'André Lhote à Jacques Rivière, du [1]3 février 1909, publiée dans La Peinture, le cœur et l'esprit, correspondance inédite (1907-1924), texte établi par Alain Rivière, JeanGeorges Morgenthaler et Françoise Garcia, Bordeaux, William Blake and co. et musée des Beaux-Arts, 1986, premier volume, p. 105.

7. André Lhote, « Le quatrième centenaire de Raphaël », NRF, n 81, 1er juin 1920, p. 928.

8. André Lhote, « Exposition Gauguin (Galerie Dru) ; Matisse (Bernheim jeune) ; Marie Laurencin (Paul Rosenberg) », NRF, n 117, 1er juin 1923, p. 968.

9. André Lhote, Réponse à l'enquête «Chez les cubistes » (IV), Bulletin de la vie artistique, n 24 (5e année), 15 décembre 1924, p. 552-554.

10. André Lhote, «André Lhote », L'Art d'aujourd'hui, 1928, p. 13-14.

11. André Lhote, « Première promenade à l'exposition », NRF, n² 288, 1er septembre 1937, p. 493.

12. Ibid., p. 493-495.

13. Ibid., p. 497.

14. Ibid., p. 498.

15. André Lhote, « La tapisserie, du XVe au XVIIe siècle », NRF, n² 297, 1er juin 1938, p. 1039-1040.

16. André Lhote, « Visite à J.M. Sert - Expositions J. E. Blanche, Bonnard, Picasso, Kisling, Lurçat, O. Des Garets, Yves Alix », NRF, n 128, 1er mai 1924, p. 645.

17. André Lhote, « Menkès - Aquarelles 1830-1930 (chez G. Bernheim) », NRF, n² 204, 1er septembre 1930, p. 431.

18. André Lhote, « De 1900 au baroquisme. Chassériau et l'inquiétude moderne », NRF, n 238, 1er juillet 1933, p. 127. 
19. André Lhote, « Gauguin et ses amis (Galerie des beaux-arts) », NRF, $n^{\circ} 248$, 1er mai 1934, p. 876.

20. André Lhote, Réponse à une interview : «L'art de M. André Lhote », Revista nuova, 6 juin 1914, p. 8.

21. André Lhote, « Le 34è salon des Indépendants », NRF, n 115, 1er avril 1923, p. 715.

22. André Lhote, « Art mural », NRF, n 299, 1er août 1938, p. 341.

23. André Lhote, «L'art italien et les artistes français », NRF, n² 262, 1er juillet 1935, p. 118.

24. André Lhote, « Les créateurs du cubisme (Galerie des beaux-arts) », NRF, $n^{\circ} 260$, 1er mai 1935, p. 788.

25. André Lhote, « Exposition Dunoyer de Segonzac », NRF, n 178, 1er juillet 1928, p. 142.

26. André Lhote, « De 1900 au baroquisme. Chassériau et l'inquiétude moderne », NRF, ${ }^{\circ}$ 238, 1er juillet 1933, p. 124.

27. André Lhote, «L'exposition des arts décoratifs », NRF, n 142, 1er juillet 1925, p. 124.

28. André Lhote, «Livres d'art », NRF, n 191, 1er août 1929, p. 289.

29. Documentation du MNAM-CCI, fonds André Lhote, chemise Lho C29, cote 5490, lettre d'André Lhote à Jacques Guenne, du 25 septembre 1934.

30. Cette revue, qui paraît de 1933 à 1935, est la troisième que publie Le Corbusier, après L'Esprit nouveau (1920-1925) et Plans (1931-1932). Voir Darlene Brady, Le Corbusier. An Annotated Bibliography, New York et Londres, Garland, 1985, p. 55.

31. Documentation du MNAM-CCI, fonds André Lhote, chemise Lho C29, cote 5490, lettre d'André Lhote à Le Corbusier, du 7 février 1935.

32. Expression de Le Corbusier lancée dans L'Esprit nouveau, en 1921, à travers la formule «La maison est une machine à habiter », à laquelle André Lhote semble implicitement répondre, en 1933, par celle d'« engins à émouvoir », pour désigner les œuvres d'art. Voir André Lhote, La peinture, le cœur et l'esprit, Paris, Denoël et Steele, 1933, préface et Yvonne Brunhammer, «Les années 1920-1930 : entre deux guerres mondiales, entre deux expositions internationales ", catalogue d'exposition Les réalismes 1919-1939. Entre révolution et réaction, Paris, Centre Georges Pompidou, 17 décembre 1980 - 20 avril 1981, p. 346

33. André Lhote, « Première promenade à l'exposition », NRF, n 288, 1er septembre 1937, p. 492.

34. Voir Laurence Bertrand Dorléac, Histoire de l'art à Paris 1940-1944. Ordre national. Traditions et modernités, Paris, Publications de la Sorbonne / Seuil, 1986, p. 209, note 1.

35. André Lhote, « Art mural », NRF, n² 299, 1er août 1938, p. 340-342.

36. Il resterait à comprendre les liens entre l'émergence de cette question dans le débat public français et l'existence, à l'échelle internationale, de pratiques d'art mural empreintes d'une dimension politique. Les relations d'étroite amitié entretenues par André Lhote avec Diego Rivera, à la fin des années 1910, à Paris, pourraient laisser supposer que Lhote est, pour le moins, au courant des expériences réalisées dans ce registre sur la scène artistique mexicaine. Mais rien n'est moins sûr, dans l'état actuel des recherches.

37. Jacques-Émile Blanche, «La profession du peintre », Encyclopédie française, tome XVII, décembre 1935, p. 72-78, cité par Blandine Chavanne et Christianne Guttinger, « La peinture décorative », dans Bertrand Lemoine (sous la direction de), catalogue d'exposition Paris 1937. Cinquantenaire de l'Exposition internationale des arts et des techniques de la vie moderne, Paris, Institut français d'architecture / Paris-Musées, 1987, p. 369 . 
38. Amédée Ozenfant, « Divorce de l'architecture et de la peinture », Encyclopédie française, tome XVI, octobre 1935, p. 70-76, cité par Blandine Chavanne et Christianne Guttinger, op. cit., p. 369.

39. Ibid., p. 364.

40. Documentation du MNAM-CCI, Fonds André Lhote, chemise Lho C29, cote 5490, lettre du 13 février 1935 à M. Charensol.

41. Voir la communication de Dominique Jarrassé au colloque cité en note 1.

42. Voir par exemple André Lhote, «L'exposition des arts décoratifs », NRF, $n^{\circ} 142$, 1er juillet 1925, p. 124 ; «Livres d'art », NRF, n 191, 1er août 1929, p. 289 ; « De 1900 au baroquisme. Chassériau et l'inquiétude moderne », NRF, n 238, 1er juillet 1933, p. 124. 43. Probablement celui de Georges Gaillard même si Lhote ne mentionne pas le nom de l'auteur. Je remercie Georg Germann pour cette précision.

44. André Lhote, « Saint-Savin "Sixtine" française », Les Lettres françaises n 33, 9 décembre 1944, p. 3.

45. André Lhote, «À propos de la rétrospective Paul Gauguin », Arts, 7 octobre 1949, p. 1 et p. 5 .

46. André Lhote, Les Chefs-d'œuvre de la peinture égyptienne, Paris, Hachette, collection "Arts du monde », 1954. Photographies de Hassia. Préface de Jacques Vandier, conservateur en chef du département des antiquités égyptiennes du musée du Louvre. Sur cet ouvrage, voir Philippe Dufieux, op. cit., p. 83-84.

47. Il restera à comprendre comment Lhote, à cet instant, prend le contre-pied d'auteurs comme Georges Bataille ou André Malraux ou d'artistes comme Tal Coat ou Raoul Ubac.

\section{RÉSUMÉS}

André Lhote (1885-1962) pratique à plusieurs reprises la peinture murale en tant que peintre mais c'est surtout en tant qu'auteur qu'il définit et défend les vertus de l'art mural et en tant que professeur qu'il encourage ses élèves à y avoir recours. Sa « théorie de l'art mural » apparaît dans ses commentaires sur des œuvres historiques ou contemporaines, dans ses leçons et conseils concrets et dans ses commentaires sur l'architecture de son temps. Il en ressort qu'il voit une forme de continuité entre des formes artistiques primitives et le cubisme. Il défend l'idée d'un « art décoratif », dévoué au mur sur lequel il s'inscrit et s'oppose à l'idée d'architecture nue.

André Lhote (1885-1962) practiced murals many times as a painter but it is especially as an author that he would define and defend the qualities of mural art and as a professor that he would encourage his students to practice it. His "theory of mural art" appeared in his comments on historical or contemporary works of art, in his lessons and concrete advices and his comments on contemporary architecture. This reveals that he saw some form of continuity between primitive artistic shapes and cubism. He would defend the idea of a "decorative art", meant to be integrated on the walls and was opposed to the idea of a bare architecture.

André Lhôte (1885-1962) beschäftigt sich zum wiederholten Male mit Wandmalerei, erstens in seiner eigenen Arbeit als Maler, dann in seinen theoretischen Schriften, in denen er den künstlerischen Wert der Wandmalerei verficht, schließlich in seinem Unterricht, in dem er seine 
Schüler immer wieder dazu anregt, sich diese Kunst anzueignen. Seine Theorie der Wandmalerei findet in seinen Erläuterungen über ehemalige wie zeitgenössische Kunstwerke Ausdruck sowie in seinen Betrachtungen über die Architektur seiner Zeit. So unterstreicht er eine gewisse unterbrochene Entwicklung von den primitiven Kunstformen bis zum Kubismus. Er vertritt die Idee einer dekorativen Kunst, ganz der Wand gewidmet, ebenso sehr, wie er sich gegen Architektur ohne Schmuck wehrt.

INDEX

Index chronologique : époque contemporaine, XXe siècle

Mots-clés : piscine, peinture murale

Keywords : swimming pool, wall painting

Schlüsselwörter : Wandmalerei, Schwimmbad

\section{AUTEUR}

\section{JEAN-ROCH BOUILLIER}

Jean-Roch Bouiller est né en 1973. Après des études universitaires d'ethnologie et d'histoire de l'art, à Lyon et à Francfort-sur-le-Main, il a rédigé une thèse de doctorat sous la direction de Françoise Levaillant intitulée « Définir et juger l'art moderne. Les écrits d'André Lhote (1885-1962) », soutenue en juin 2004 à l'université Paris I Panthéon-Sorbonne. Lauréat du concours de l'École nationale du patrimoine en 1997, il est depuis juillet 2000 conservateur des monuments historiques à la direction régionale des affaires culturelles de Provence-Alpes-Côte d'Azur. Il est par ailleurs chargé de cours à la faculté d'histoire de l'art de l'université de Provence, membre correspondant du centre André Chastel (UMR 8150, DAPA-CNRS-université Paris IV), et membre du comité français d'histoire de l'art (CFHA). Il a publié plusieurs articles sur André Lhote, sur l'art contemporain ou sur le patrimoine. Il a co-organisé en mars 2006 avec Dario Gamboni et Françoise Levaillant des journées d'études sur les bibliothèques d'artistes aux $\mathrm{XX}^{\mathrm{e}}$ et $\mathrm{XXI}^{\mathrm{e}}$ siècles (actes à paraître). 